\title{
Thomas J (2016) Les Incas et la déformation intentionnelle du crâne. Un marqueur social, culturel, ethnique et religieux - Presses universitaires de Nancy - Éditions universitaires de Lorraine, ISBN : 978-2-8143-0278-5, 136 pages, $12 €$
}

\author{
S. Natahi \\ (C) Société d'Anthropologie de Paris et Lavoisier SAS 2019
}

Cet ouvrage de Jérôme Thomas est composé de 136 pages au cours desquelles l'auteur aborde la thématique des pratiques de déformations intentionnelles du crâne. L'ouvrage s'appuie sur une littérature abondante puisque l'on compte en tout deux cent seize références écrites en six langues distinctes (Allemand, Anglais, Espagnol, Français, Latin et Portugais).

L'ouvrage est divisé en plusieurs chapitres (au nombre de sept) dont les premiers sont essentiellement consacrés à des considérations généralistes sur la nature de ces pratiques, leur perception au sein de la société moderne ainsi qu'à un historique de la recherche sur le sujet. Le troisième chapitre est quant à lui consacré à un commentaire des sources historiques et ethnohistoriques les plus remarquables qui firent mention de cette coutume. L'auteur offre au lecteur un tour d'horizon des pratiques de déformations intentionnelles du crâne à travers les cinq continents, depuis ses formes d'expression les plus anciennes jusqu'à des périodes plus récentes. Ce travail de documentation conséquent nous permet d'en mesurer pleinement le caractère ubiquiste et rend parfaitement compte de la grande diversité des techniques employées. Il est à noter qu'il s'agit là d'une première synthèse non exhaustive publiée en langue française. Suite à cela, l'auteur va orienter et limiter son propos aux cadres des Amériques.

Dans un réel intérêt porté à la pratique en tant que telle et non à sa résultante finale (i.e. la forme du crâne), l'auteur a fourni un important travail d'archives et exploré un grand nombre de sources historiques, ethno-historiques ou ethnographiques lesquelles constituent de précieux témoignages pour qui souhaite se pencher sur les pratiques de déformation du crâne dans les cultures précolombiennes du continent sud-américain. On pourra tout de même trouver dommage que parmi les illustrations de l'ouvrage ne figurent pas les deux illustrations de Guamán Poma de Ayala [1] qui constituent deux uniques témoignages graphiques de la période coloniale, montrant des enfants emmaillotés dans des ber-

S. Natahi

UMR 5199 PACEA, Université de Bordeaux, France ceaux et coiffés de ce qui semble s'apparenter à un bonnet ; possible appareil céphalique employé à des fins de modelage de la tête de l'enfant.

La grande force de l'ouvrage, outre la richesse de ses références, réside dans l'effort de son auteur à fournir une lecture plus approfondie et complexe que celles souvent évoquées dans les sources ethnohistoriques, lesquelles attribuent à ces coutumes des considérations un peu trop simplistes, interprétées à l'aune des codes culturels de ceux qui en sont les témoins et rapporteurs directs. De telles coutumes y sont très souvent perçues comme véhiculant des valeurs identitaires ou encore comme porteuses de croyances religieuses ou de distinctions d'ordre social. La dimension utilitaire, certes moins courante, est aussi parfois évoquée : le front plat facilitant, selon certains auteurs, le port de charges lourdes. En effet, nombre de chroniqueurs contemporains de la Conquête à l'instar de Bartolomé de Las Casas, Bernabé Cobo, Juan Ulloa-Mogollón, appréhendent la déformation intentionnelle du crâne comme un vecteur d'affirmation d'une identité et d'une appartenance culturelle que le pouvoir inca aurait artificiellement maintenu afin d'identifier au mieux le lignage de chacun de ses sujets. En se penchant sur une série de témoignages ethnohistoriques de ce type, l'auteur, lui, s'attache à la réalisation d'un véritable travail historique ; celui d'une lecture critique des sources qui cherche à nuancer ce type d'interprétations en mettant en évidence les rapports bien plus complexes qui sous-tendent l'expression de ces pratiques.

Même si ce travail a pour support principal des documents historiques coloniaux, on pourra regretter l'absence d'une partie abordant brièvement la chronologie andine précolombienne. Ce chapitre aurait eu le mérite de mettre en contexte les transformations politiques, territoriales et culturelles qui intervinrent au cours de l'Horizon récent (14001532 apr. J.-C.), période qui marque l'expansion de l'Empire inca. Il aurait apporté au lecteur, parfois peu familier à l'histoire complexe des populations précolombiennes andines, un regard plus large sur la mise en place d'un État composé d'une mosaïque de cultures réunies sous son joug. 
Par ailleurs, l'auteur met judicieusement en avant, dans un premier temps, les rapports indissociables qui existent entre les divers éléments de parure corporelle. En effet, n'étudier qu'un seul de ces aspects au détriment des autres ou en dépit de leur considération ne saurait être pertinent :

"Elles [les têtes déformées] s'inscrivent dans un système global ou la déformation n'est qu'un objet parmi d'autres. [...] De plus, les coiffures et la symbolique de la chevelure doivent être étroitement associés à cette coutume pour en comprendre tout le sens. Déformations, bonnets, coiffures et chevelure doivent être analysés ensemble. » (p. 72)

Dans un second temps, l'auteur s'attache également à souligner les considérations spirituelles attribuées à la tête en tant qu'élément corporel canalisant l'énergie vitale. Il montre son imprégnation de croyances religieuses fortes qui se seraient manifestées au travers de ces manipulations rituelles (déformation du crâne, coiffure, apposition de coiffes) intervenant dès la naissance mais aussi lors de rites de passage d'un âge à un autre.

L'acte de déformer le crâne participerait alors d'un système complexe et indivisible dont la pensée cosmogonique des peuples précolombiens des Andes serait un fondement. Il semble très vraisemblable que ces pratiques relèvent d'un système global d'ornementation, codifié et sous-tendu alors par des considérations multiples (cosmogoniques, identitaires, rituels) et interpénétrées.

Mannheim, Davis et Velasco dans un ouvrage récemment édité par Vera Tiesler et María Cecilia Lozada, dressent d'ailleurs un constat similaire et estiment que ces pratiques ne peuvent être considérées qu'à l'aune de l'ontologie sociale des peuples andins [2]. Dans ce chapitre d'ouvrage, ces mêmes auteurs se penchent sur deux populations de la vallée de Colca (située dans la partie méridionale du haut plateau péruvien) : les Collaguas et les Cavanas. Les auteurs évoquent le fait que ces deux groupes présentaient des pratiques de déformation différentes (de type annulaire pour les Collaguas et tabulaire pour les Cavanas) précédant toutes deux l'avènement de l'État inca et qui se maintinrent par la suite [2-3]. Ces deux groupes, outre leur différenciation linguistique, le premier principalement de langue Aymara et le second de langue Quechua, se distinguaient également par l'occupation de l'espace ; les Collaguas occupant les hautes terres de la vallée tandis que les Cavanas en peuplaient les basses terres [4]. Dans cet exemple, les auteurs parviennent à une conclusion assez similaire à celle de Jérôme Thomas et montrent que la différence de pratiques de déformation crâniennes participerait de concert avec des différenciations linguistiques mais aussi écologiques. La compréhension des facteurs motivant ces pratiques dans les populations andines ne saurait donc avoir de sens sans une prise en compte des considérations sociales, culturelles, religieuses ou écologiques de ces groupes humains.

On conçoit alors toute l'importance que le pouvoir inca, conscient du profond enracinement de ces pratiques au sein d'un système de croyances séculaires, a attaché à leur codification et leur législation. Leur respect et leur maintien par les peuples qu'il fédère figurent non seulement comme un vœu d'allégeance mais constitue également un instrument au service du pouvoir dans l'objectif d'identifier l'appartenance culturelle de chacun de ses sujets. Comme le montre Jérôme Thomas, les Espagnols avaient bien compris également le caractère rituel de ces pratiques mais n'en avaient peut-être pas mesuré toute la profondeur. Dans le dernier chapitre de l'ouvrage l'auteur se penche ainsi sur les diverses mesures mises en œuvre en vue de leur prohibition du fait de leur nature idolâtre. L'auteur y évoque les vaines (bien que nombreuses) tentatives d'interdiction qui dénotent parfaitement la prégnance de ces pratiques au sein des cultures andines précolombiennes.

On pourra sans doute déplorer l'absence d'une prise en compte des données archéologiques qui auraient apporté un regard diachronique sur l'évolution des pratiques de déformations crâniennes entre l'Intermédiaire récent (1000-1400 apr. J.-C.) et l'Horizon récent, et sans doute donné aux hypothèses de l'auteur une profondeur plus importante. Il aurait été par exemple intéressant de prendre en considération l'hypothèse proposée par Torres-Rouff, laquelle associe conjointement l'homogénéisation des pratiques de déformations crâniennes à la complexification des formes d'organisations sociales [5]. L'ouvrage de Jérôme Thomas n'en demeure pas moins important en ce sens qu'il constitue une première synthèse en langue française sur ce type de pratiques. Les sources documentaires y sont abondantes. L'auteur y a le mérite d'aborder les pratiques de déformation intentionnelles du crâne chez les populations andines dans toute leur complexité, évoquant ainsi la pluralité des caractères qui soustendent leur expression.

\section{Références}

1. F Guamán Poma de Ayala (1613) Nueva Corónica y Buen Gobierno, Fac-similé [1989], Institut d'Ethnologie 1168 p

2. B Mannheim, AR Davis, MC Velasco (2018) Cranial Modification in the Central Andes Person, Language, Political Economy. In V Tiesler, MC Lozada (eds.) Social Skins of the Head Body Beliefs and Ritual in Ancient Mesoamerica and the Andes, pp. 223-33. University of New Mexico Press, Albuquerque

3. MC Velasco (2016) Mortuary Tradition and Social Transformation during the Late Intermediate Period (AD 1100-1450): A Bioarchaeological Analysis of Above-Ground Burials in the Colca Valley, Peru. Unpublished PhD dissertation, Department of Anthropology, Vanderbilt University, Nashville, $321 \mathrm{p}$

4. Wernke (2006) Collagua "Eco-Logistics": Intermediate Elites and Hybrid Community Structures in the Colca Valley, Peru. In Elson $\mathrm{CM}$ and Covey RA (eds.) Intermediate Elites in Pre-Columbian States and Empires, University of Arizona Press, Tucson, pp. 175-210

5. Torres-Rouff C (2003) Shaping Identity: Cranial Vault Modification in the Pre-Columbian Andes. Unpublished PhD dissertation, Department of Anthropology, University of California, Santa Barbara, $414 \mathrm{p}$ 